\title{
Cloud Computing: A Detailed Relationship to Grid and Cluster Computing
}

\author{
Ikechukwu Nwobodo
}

\begin{abstract}
Cloud computing continues to maintain a rapid growth while constituting a viable alternative to conventional computing. Cloud computing is an emerging business computing model that enable users access to IT resources via internet infrastructure at any PC connected node across the globe without hardware constraints. Cloud computing concept is a development trend based on several successful researches in computing areas such as virtualization, grid computing, cluster computing and distributed computing. Cloud, cluster and grid computing share some common features towards achieving utility or service oriented computing although marginal differences exist on their processes and approaches in many ways. This paper will be exploring the evolutionary relationships in terms of features and component of cloud computing technological innovation in comparison to cluster and grid computing.
\end{abstract}

Index Terms-Cloud computing, grid computing, cluster computing, utility computing.

\section{INTRODUCTION}

The use of a high performance computing (HPC) was previously restricted to organisation that has financial capacity to afford exorbitant and highly dedicated supercomputer at the period. Hence there was an urgent requirement for high performance computer in relatively small scale at a comparatively lower cost which then, prompts to a more computational approach as cluster computing. The advent of cluster computing was orchestrated by a varying number of academic projects such as Berkerly NOW, HPVM and Beowulf [1]. However, the emergency of internet and the availability of sophisticated computers including low cost commodity components as high speed networks technologies have dramatically changed how we operate computing. Grid computing originated from academia conception during 1990s with the objective of enabling user's at times when their local systems are busy to remotely utilize idle computing capabilities at other computing centres. At early inception, it was basically referred as compute grid and adherently commands little audience. Consequently, after many years of concerted efforts in development became an efficient, effective means of computing resource sharing and problem solving solution in multi institutional dynamic virtual environment. Subsequently, cloud computing model became popular at the dire end of 2007 [1]. It introduces a new meaning to off

Manuscript received December 10, 2014; revised February 3, 2015.

Ikechukwu Nwobodo is with School of Architecture, Computing and Engineering University of East London, Dockland Campus, London, United Kingdom (e-mail: i.nwobodo@yahoo.com). premises and distributed computing perception, by providing to users pool of computing resources in utility consumption forms accessible via internet on demand. The process of cloud resource distribution and allocation makes it easier for enterprises to access and use cloud computing resources such as network, storage, and application servers without requirement for huge upfront investment on infrastructure provided, thereby strengthening or allowing concentration on other business critical development. Cloud computing resources are dynamically accessed or requested on demand without prior reservation and in essence helps to eliminate issues of resource over-provisioning resulting to improved utilization of resources [2], [3].

\section{THREE MODELS OF COMPUTING}

The three computing models: cluster, grid and cloud computing will be explained in this section.

\section{A. Cluster Computing}

Super computer was at fore front in the field of computing for many years. In view of enormous issues faced in science, business and engineering area at the time which supercomputer failed to solve effectively. However, cluster computing was the alternative used to replace them with the objective of eliminating these issues, more importantly, considering how cluster computing presents opportunity of access to potentially low cost and massive computing power at the time. A cluster computing is a group of inter-connected stand-alone, distributed or parallel computers dedicated to working together and sharing computing workload as a single integrated system resource [1], [4], [5]. All nodes involved in cluster computing are interconnected using gigabit Ethernet as a high speed network link, working together, share and executing compute exhaustive task as a single computing entity that would practically unrealizable using single computer as shown in Fig. 1 below. The purpose of cluster use is mainly for high availability compute, fault tolerant, load balancing and redundancy. The use of cluster for high availability is to ensure continuous services as it helps to maintain redundant nodes in event of system component failure. System performance is improved because when a node fails, there are other standby nodes which will take over the task thereby eliminating single point of failure in network. Moreover, any fault in one component only affects the level of cluster performance power but not its availability. Other factors necessitating the use of cluster include applications requiring high computation in terms of throughput, response time, memory, especially real time application. Cluster computing enable high computation by 
adoption of parallel programming which entails use of multiple processors concurrently for single or several issues. Realistically, user's perspective means they are multiple computers that functions as one virtual machine. The user compute request are received and simultaneously distributed between all standalone computers in cluster. The outcome of this structure results in a balanced computational task among different machine contributing to the performance of the cluster model. Clusters are known to be used often mainly for computational purpose rather than activities based on IO [1], [4].

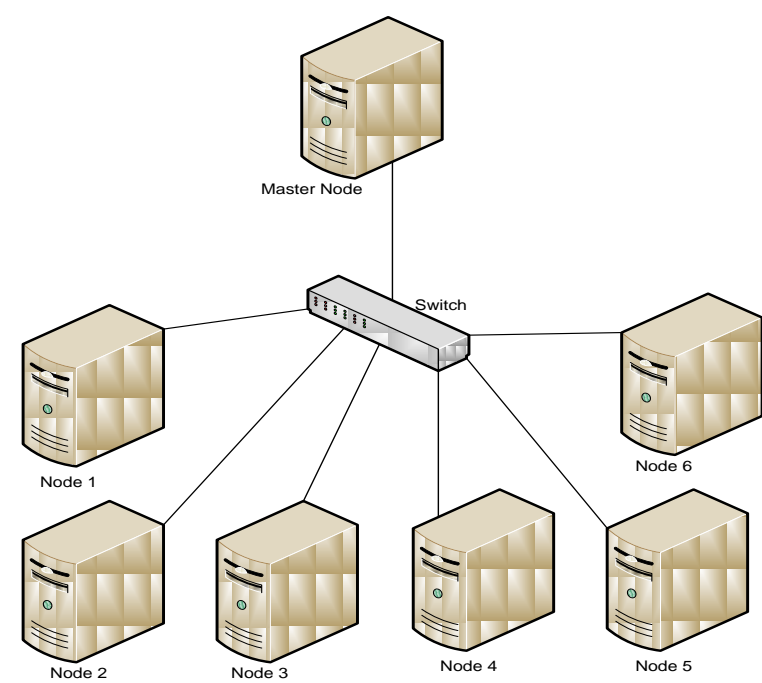

Fig. 1. Cluster computing.

\section{B. Grid Computing}

Grid computing emerges in mid 1990 for the purpose of solving large scale computation problems by combining multiple computing resources from various clusters of autonomous, heterogeneous, geographically distributed systems as shown in Fig. 2 below. The term was originally coined as a description of computing technology that will enable users to access computing powers on demand [6]. Grid computing is defined as a type of distributed systems that allow the aggregation, selection, sharing of clusters of geographically dispersed autonomous resources dynamically at runtimes based on their performance, availability, cost and user's quality of service needs [4], [5]. The innovative idea behind grid computing is to allow aggregation of computing resources from multiple locations to resolve issue that can't be resolved using the processing capability of a single computer. Conceptually, grid computing initiatives are analogous in utility and form to electrical grid as in an electrical power grid, the wall outlet enable us to connect resource infrastructure that generate and distribute electricity. However, when we access the electrical grid, we don't deem it important to know how the electrical current is being connected to our home or where the plant is located and this is similar to user resource access in computing gird [2], [4]. The main grid computing strategy is utilizing middleware to manage diverse computing resources across multiple networks by sharing and allocating pieces of program task between several computers at same time enabling them to act or function as a single virtual entity. The two distinct related goals of grid are providing users remote access to IT resources and same time aggregating system processing power. Grid main resource is processor although also include application, data storage system, sensors etc. Grid size differs from a small workstation of a network computer within an enterprise to a large collaboration across many organization networks. There are two important outcomes to grid which is the Open Grid Service architecture and the Globus Toolkit.

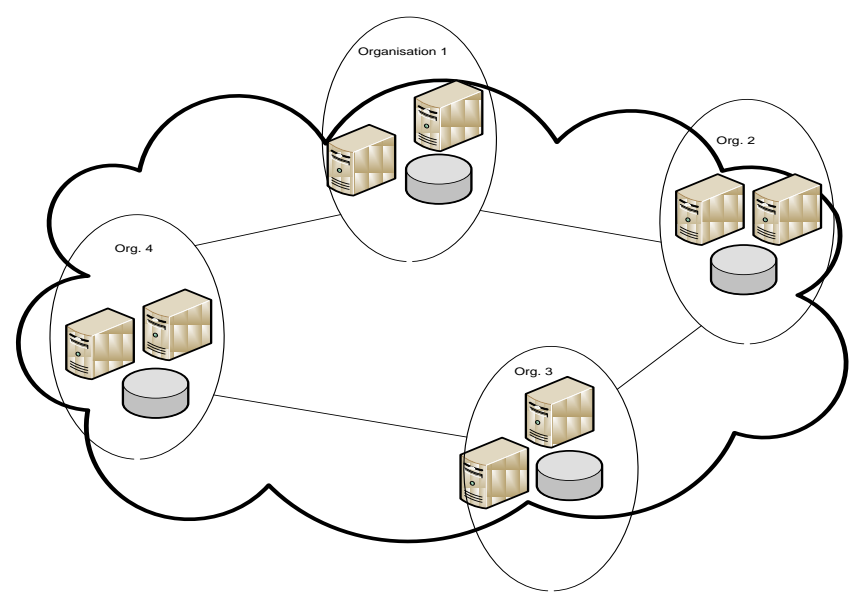

Fig. 2. Grid computing.

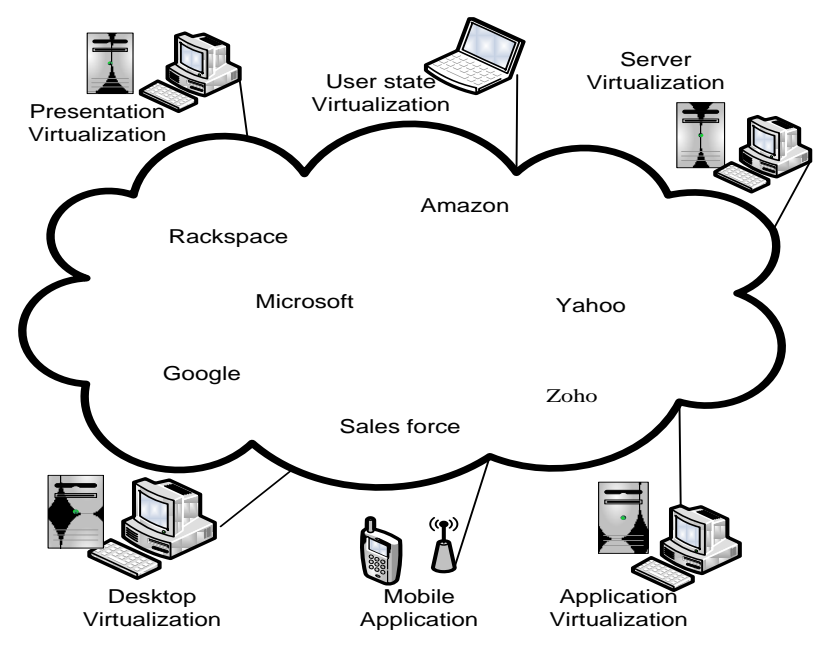

Fig. 3. Cloud computing.

\section{Cloud Computing}

Cloud computing is a novel computing paradigm based on advancement of virtualization technology that provides huge dynamic, scalable pools of virtual IT resources on demand as a service via internet technology. This is a new computing concept that magnificently usher a modern and efficient transformational meaning to off-premises, cluster, grid and distributed computing. Cloud computing refers to a representation of IT resource such as applications, storage and system software's delivered by cloud service provider through the web based on user requirements as depict in Fig. 3 below [1], [4]. According to Buyya et al. [5] a cloud is a type of parallel and distributed system consisting of a collection of inter-connected and virtualized computers that are dynamically provisioned and presented as one or more unified computing resource based on service-level agreements, negotiated, time-honored between consumers and cloud service providers. The infrastructure provisioned in cloud includes scalable resources as network, storage and 
application that can be elastically deployed within a short time thereby reducing management overhead to cloud provider and unnecessary administrative time to end users [3], [7]. Cloud computing to consumers entails pay per use on demand mode of shared IT resources, accessed via internet from any end user computers anytime any where across the Globe without limitation to resource requirement or hardware demands. Cloud resources are visible to users, meaning users can dynamically lease and use cloud resources without the need of knowing where and how cloud resources exist. Cloud computing deployment types include: public, private, hybrid and community cloud while cloud computing services consist of Infrastructure as a service (IaaS), Software as a service (SaaS) and Platform as a service (PaaS) [3], [7], [8].

\section{Cluster Computing Project, ApPlicAtion AND TOOLS}

This section will be used to outline some important applications, projects and tools in cluster computing environment.

\section{A. Cluster Computing Projects}

Condor [1], [9] is a multipart project with key activities in five areas such as complex software engineering, distributed computing research, scientific computing participation, productive environment maintenance and student education

Shared Research Computing Services (ShaRCS) [10] is vital cluster project primarily designed to characterize, exhibit and quantify how storage and shared computing clusters based in various data centre locations can offer critical services to investigators

\section{B. Cluster Computing Applications}

Hadoop [11] is a framework based on open - source used for running application data intensive within a processing large cluster developed from commodity hardware. It provides transparent application data motion and reliability. The framework implements computational paradigm called Map/Reduce. A lot of users utilize Hadoop clustering to analyze search patterns of customers for possible advertising targets. There are other application in this category used for indexing and filtering web listings, image searches for facial recognition algorithms in massive database

Multi Applications: Cluster computing were heavenly used in solving issues involving weather modeling, processing images, automobile crash simulations, life sciences, data mining, astrophysics, electromagnetic, aerodynamic and simulations. Clusters are used as a platform for application data mining that requires compute and data intensive operations. A lot of enterprises use the application for realization of high availability for backup especially in the banking sector. Internet services sites such as hotmail, database are also host in the cluster environments.

WRF (Weather Research and Forecast) [12] is a combined project by many institutions to design and model collaboratively next generation regional forecast model, data simulation system for atmospheric research and numerical weather prediction.

\section{Cluster Computing Tools}

Condor [1], [9] is a specialized resource and job management system developed specifically for compute intensive jobs. The tool enables mechanism for job management, priority scheme, policy scheduling, resource management and monitoring. According to the process, jobs are submitted by users and the tool chooses where and when job will be run based on set of policy, then their progress are monitored and as a result inform users as soon as job is completed.

Nimrod [13], [14] is a cluster tool for parametric computing capable of providing a simple declarative parametric language modeling towards expressing a parametric experiment. The use enable easy development task for a parametric computing and utilizing the Nimrod runtime system to run, submit and finally collect various multiple cluster nodes result.

MPI and OpenMP [15] is a message passing libraries that enable well rated way of passing data between distributed memories executing process. It contributes to a high performance of collection of single cluster nodes. The de factor standard for parallel programming on cluster is MP1 and it consists of high quality set of libraries function to perform both collective and point to point communication between parallel tasks

Flexi-Cluster and VERITAS: The Flexi-Cluster is specifically a simulator for a particular compute cluster while the VERITAS is a simulator cluster for servers.

PARMON: This is a cluster tool that specifically at three different levels enables system resource monitoring including their activities i.e., node, components and system. The C-DAC PARAM 10000 supercomputer which is a 48 Ultra-4 workstation cluster powered by Solaris is monitored using the tool.

\section{GRID COMPUTING, Project, APPLICATION AND TOOLS}

This section will be used to outline some important applications, projects and tools in grid computing environment.

\section{A. Grid Computing Projects}

EGI-InSPIRE [16] (Integrated Sustainable Pan-European Infrastructure for Researchers in Europe) project: Commenced in 2010 and directly funded by European Commission for four years as a joint efforts comprising over 50 institutions in more than 40 countries. The objective is to establish a sustainable Grid Infrastructure in Europe by joining new distributed computing Infrastructure together which includes: supercomputing systems, clouds desktop grids for the benefits of users in European Research Area communities. The crucial aim is to establish an environment for European scientist including their international partners with an efficient and reliable infrastructure that will help them succeed in their mega, high level data analysis.

Major Grid Projects: Other notable grid project includes NASAs Information Power Grid, National Technological Grid, GriPhyN, Particles Physics Data Grid and the European Data Grid, Globus [17], [18] is open source grid software specifically designed to eliminate issues relating to 
distributed resource sharing problems. Globus DemoGrid was the first to be released as a tool for the development of instructional grid environment capable of being deployed using virtual machines on a physical resource or cloud environments. The objective of the project was to provide an easy and accessible environment with different grid tools, without the need of installing the tools or requiring an account on active grid.

\section{B. Grid Computing Application}

Drug Discovery Grid (DDGrid) [1], [19]: The objective of the project is developing platform for combined efforts towards drug discovery via $\mathrm{P} 2 \mathrm{P}$ and grid computing technology. It is expected to solve issues of mega computation and data intensive application in area of molecular biology, chemistry and medicine using grid middleware.

Mammo Grid [1], [19] is a medical grid application relied mainly as service oriented architecture. The objective is primarily to deliver a set of evolutionary prototype to show mammogram analysts, radiologist specialist particularly working in breast cancer screening that can utilize the grid infrastructure information for potentially resolving basic issues of image analysis.

Geodise [19] is used mainly to provide a Grid-based integration framework for data intensive multidisciplinary design optimization tasks and computation

\section{Gird Computing Tools}

Condor-G [20] is a Globus and Condor project work representation which allows the large collection of resource utilization spanning across multiple networks in such a form as if they belong only to single user domain or personal network. The use of protocols for standardized access and inter domain communications to remote batch systems comes from Globus while concerns such as error recovery, job submission, and friendly execution environment creation comes from Condor.

GRID computing and Business (Gridbus) [21] is a cluster and grid toolkit middleware technology project associated with their design and development for service oriented computing. The toolkit enables end to end services to aggregate or lease distributed resource services based on their capability, availability, cost, QoS requirements and performance.

Paradyn: Paradyn is used for analysis as well as support for experimental management performance via qualitative technique of comparing many experiments performance diagnosis according to dynamic instrumentation. The performance analysis is automatically executed while experiment is manually set up.

Globus [18] is an open source software toolkits that enable computational grid and grid based application construction across geographical boundaries

Legion: Legion is an object based metasystem that enable data management, site autonomy, transparent core scheduling, fault tolerance and in addition a middleware with broad array of various security options.

Nimrod-G [14] Nimrod-G uses Globus middleware service for dispatching job over computational grid and at same time for dynamic resource discovery. These enable engineers and scientist to transparently organize program and data in remote sites as well as run the program on a particular element of a data set specifically on difference machine and at last assemble all results to a user site from the remote site.

Simulators [14]: There are other simulators used in grid computing such as Optorsim, ZENTURIO, Chicsim and Gridsim etc.

\section{Cloud Computing Project, Application And Tools}

This section will be used to outline some important applications, projects and tools in cloud computing environment.

\section{A. Cloud Computing Project}

CESWP (Cloud-Enabled Space Weather Platform) [22]: The aim of the project is to use cloud computing flexibility and opportunity to space weather physicist. The objective is to overcome obstacle and make it easier for physicists to conduct their respective science such as develop space weather models, enable collaboration with other scientist, undertake simulation tasks, produce virtualization and allow provenance

CERN: The European for Nuclear Research is developing a large scale cloud computing generally to be used in distributing data to scientist across the globe specifically as part of LHC project.

TCloud [23] project is for development of advanced cloud computing infrastructure prototype capable of delivering a new form of secure, private, resilient computing storage that will be scalable, simple and cost efficient

OpenNebula [24] is an open source project which has successfully released notable advance, flexible, enterprise ready cloud computing management tool. The goal is to develop advanced, well scalable, adaptable cloud computing software toolkit. The aim is to simplify OpenNebula management cloud instance, including fault tolerance functionality to ensure cloud uptime, new security functionality, images and templates enhanced managements, federation of data centre and multi-tier architecture enhanced support.

UCI (Unified Cloud Interface) [25]: The Cloud Computing Interoperability Forum (CCIF) is a body working for the development of standard API. They proposed UCI project to be used by all cloud computing service provider to solve issues of cloud interoperability.

\section{B. Cloud Computing Application}

Cloudo [1], [19] is a free computer that resides in the internet via web browser. This can be utilized to access music's, documents, movies, photos and other important files from any Mobile phones or PC.

Panda Cloud Antivirus [1], [19] is the first free antivirus software from cloud computing. The application is known to occupy only a little system resources and has history of regular updates normal, in addition makes use of combined intelligent servers for potential fast detection, runs offline protection and encompasses a simple user interface. 
Library Resource: In view of cloud computing rapid expansion and current establishment of public cloud within a lot of university libraries, there are indications that such move will help keep library resources and advance satisfaction of users. Presently, there are Online Public Access Catalogue (OPAC) and Inter library Loan (ILL) services, there are difficulties posed for shared resources access via uniform access platform which can be eliminated by generally adopting cloud computing solution for library services.

RoboEarth [1], [19] project is led by Eindhoven University of Technology, Netherlands, a European project for development of WWW for robots. It comprises of a mega database where objects, environment and tasks information can be shared by robots. Also, at AORO A-Star Social Robotics Laboratory Singapore, a cloud computing infrastructure that enable generation of 3-D maps by robots in their environment as much faster than they could using their normal onboard PC.

\section{Cloud Computing Tools}

CloudSim and CloudAnalyst [1]: The tool is valuable to developers as it is used to evaluate large scale cloud application requirement in respect to user workloads and computing server's geographical distribution. The later was developed to assist developers in having insight on ways to adopt for application distribution within cloud infrastructures including value added services while the former was modeled for the objective of studying application behaviors within several deployment configurations.

Zenoss [26] is a single integrated tool used for entire IT infrastructure monitoring, irrespective of deployed location in cloud both virtual and physical. It provides management to cloud deployments, virtual devices, storage, servers etc.

Cloudera [27] is an open source Hadoop software framework much used for cloud computing deployment based on its flexibility with data intensive queries and other major tasks. It also enables exploration of complex, non relational data particularly in its native form.

Spring Roo: This is a modern tool for application development, with combined strength of Google Toolkit (GWT) that allows developers in enterprise production environments to model rich browser application.

\section{Cluster, GRid AND Cloud Computing Comparison}

Looking at wider viewpoint, cluster, grid and cloud computing concept seem to share analogous features in many ways. This section throws a comprehensive picture to differentiate in various perspectives by clearly via tabular form highlighting each comparable area as displayed in Table I below.

TABLE I: CLUSTER, GRID AND CLOUD COMPUTING COMPARISON

\begin{tabular}{|c|c|c|c|}
\hline & Clusters & Grids & Clouds \\
\hline Ownership & Single Ownership & Multiple Ownership & Single Ownership \\
\hline Service Pricing & Limited & Private or public assigned & Utility /large user discount \\
\hline Virtualization & Half & Half & Yes \\
\hline Resource Management & Centralized Resource & Distributed Resource & Both \\
\hline Scalable Size & $100 \mathrm{~s}$ & $1000 \mathrm{~s}$ & 100 to $1000 \mathrm{~s}$ \\
\hline Standardized & Yes & Yes & No \\
\hline Interoperability & Yes & Yes & Not full \\
\hline $\begin{array}{l}\text { Interconnection } \\
\text { network/speed }\end{array}$ & $\begin{array}{c}\text { Dedicated high end with } \\
\text { low latency \& high } \\
\text { bandwidth } \\
\end{array}$ & $\begin{array}{l}\text { Mostly internet with high latency } \\
\text { a \& low bandwidth }\end{array}$ & $\begin{array}{l}\text { Dedicated high end with low latency and high } \\
\text { bandwidth }\end{array}$ \\
\hline Self service & No & Yes & Yes \\
\hline Single system image & Yes & No & Yes/Optional included \\
\hline Multi-tenancy & No & Yes & Yes \\
\hline Service negotiation & Limited & Yes, SLA based & Yes, SLA based \\
\hline Discovery of membership & Membership services & $\begin{array}{l}\text { Decentralized info. Services and } \\
\text { centralized indexing }\end{array}$ & Membership service discovery \\
\hline Operating System & Windows/Linux & $\begin{array}{c}\text { Any standard (dominated by } \\
\text { Unix) }\end{array}$ & A hypervisor(VM)on which multiple OS run \\
\hline Application drivers & $\begin{array}{l}\text { Business, data centres, } \\
\text { enterprise computing }\end{array}$ & $\begin{array}{l}\text { Collaborative scientific \& high } \\
\text { throughput applications }\end{array}$ & $\begin{array}{c}\text { Web App. Content Delivery, dynamically } \\
\text { provisioned }\end{array}$ \\
\hline Standards/interoperability & $\begin{array}{c}\text { virtual interface } \\
\text { Architecture (VIA) }\end{array}$ & Some Open Grid Forum & Web Services (SOAP and REST) \\
\hline Scalable & No & Half & Yes \\
\hline Failure management & $\begin{array}{c}\text { Limited (often failed } \\
\text { task/application \& restarted }\end{array}$ & $\begin{array}{c}\text { Limited (often failed } \\
\text { task/application restarted }\end{array}$ & $\begin{array}{l}\text { Failover, content replication, VM migration from } \\
\text { a node to another supported }\end{array}$ \\
\hline Capacity & $\begin{array}{c}\text { Stable \& guarantee } \\
\text { capacity }\end{array}$ & Varies, but high capacity & Provisioned on demand capacity \\
\hline Security & $\begin{array}{c}\text { Traditional } \\
\text { login/password-based }\end{array}$ & $\begin{array}{l}\text { Public/private pair based } \\
\text { authentication and mapping a user } \\
\text { to an account }\end{array}$ & $\begin{array}{l}\text { Each user /application is provided with a virtual } \\
\text { machine }\end{array}$ \\
\hline Privacy & $\begin{array}{c}\text { Medium level of privacy } \\
\text { depends on user privileges }\end{array}$ & Limited support for privacy & $\begin{array}{c}\text { Hi security/privacy is guaranteed. Support for } \\
\text { setting per-file access control list (acl) }\end{array}$ \\
\hline Population & Commodity computers & $\begin{array}{c}\text { High end systems (clusters, } \\
\text { servers) }\end{array}$ & $\begin{array}{c}\text { Commodity PCs, high end servers network } \\
\text { attached storage }\end{array}$ \\
\hline End user presentation & $\begin{array}{c}\text { Presented as a dynamic and } \\
\text { diversified system }\end{array}$ & Presented as a single system image & Presented as a self-services based usage model \\
\hline
\end{tabular}




\section{DisCUSSION ON CLUSTER, GRID AND CLOUD COMPUTING}

Computing models have tremendously undergone several development levels as reiterated in section two of this paper. In order to have a better understanding, a comprehensive comparison of cluster, grid and cloud computing based on different perspective was presented in Table I above and some parameters used include: ownership type, service pricing, virtualization etc. Conceptually, their feature seems related although minor differences exist. For example, the whole model has some certain level of scalable sizes and virtualization etc. However, they differ in a lot of ways in term of resource management, standardization, self service, multi-tenancy etc. Cluster computing resources are administratively located and managed in a single domain while grid resources are distributed geographically across multiple administrative domains with their own respective management goals and policies. Contrarily, cloud computing technology possesses major characteristics of both cluster and grid computing, in addition to its own capabilities and attributes such as extensive support for virtualization, dynamically provisioning of computing resources as utility form of consumption i.e., storage, network and application on demand via web interface to users without reference to hosted infrastructure.

\section{CONCLUSION}

Cloud computing is a new computing paradigm which has evolved from the advancement of virtualization technology in recent times. The current popularity being attracted by cloud computing from divergent audience can be attributed to its architectural design, development models and services specifically modeled as a target towards utility based consumption and technology. This paper has been used to present an evolutionary relationship and marginal differences of cloud computing technology to other computing models such as Grid computing and Cluster computing, more especially their various applications, tools and projects relevant to its technological growth.

\section{REFERENCES}

[1] N. Sadashiv and S. D. Kumar, "Cluster, grid and cloud computing: A detailed comparison," in Proc. the 2011 6th International Conference on Computer Science and Education (ICCSE), 2011, pp. 477-482.

[2] S. Zhang, X. Chen, S. Zhang, and X. Huo, "The comparison between cloud computing and grid computing," in Proc. the 2010 International Conference on Computer Application and System Modeling (ICCASM), 2010, vol. 11, pp. 11-72.

[3] I. Nwobodo, H. Jahankhani, and A. Edoh, "Security challenges in the distributed cloud computing," International Journal of Electronic Security and Digital Forensics, vol. 6, no. 1, pp. 38-51, 2014.

[4] K. Kaur and A. K. Rai, "A comparative analysis: Grid, cluster and cloud computing," Lecture Notes in Computer Science, pp. 341-342, 2010.

[5] R. Buyya, C. S. Yeo, S. Venugopal, J. Broberg, and I. Brandic, "Cloud computing and emerging IT platforms: Vision, hype, and reality for delivering computing as the 5th utility," Future Generation Computer Systems, vol. 25, no. 6, pp. 599-616, 2008.
[6] I. Foster, Y. Zhao, I. Raicu, and S. Lu, "Cloud computing and grid computing 360-degree compared," Grid Computing Environments Workshop, pp. 1-10, 2008.

[7] G. Suciu, S. Halunga, A. Apostu, A. Vulpe, and G. Todoran, "Cloud computing as evolution of distributed computing-A case study for slapos distributed cloud computing platform," Informatica Economica, vol. 17, no. 4, pp. 109-122, 2013.

[8] A. Khalid and M. Shahbaz, "Cloud computing technology: services and opportunities," Pakistan Journal of Science, vol. 65, no. 3, 2013.

[9] D. Thain, T. Tannenbaum, and M. Livny, "Distributed computing in practice: The Condor experience," Concurrency and Computation: Practice and Experience, vol. 17, pp. 323-356, 2005.

[10] UC. (2014). UC Shared Research Computing Services. [Online] Available: http://srcs.ucop.edu/pilot.php

[11] Apache. (2014). Welcome to Apache Hadoop. [Online] Available: http:// hadoop.apache.org/.

[12] G. Shainer, T. Liu, J. Michalakes, J. Liberman, J. Layton, O. Celebioglu, and D. Cownie, "Weather research and forecast (WRF) model performance and profiling analysis on advanced multi-core HPC clusters," Global Forecast System, 2009.

[13] MeSsAGEge Lab. (2011). [Online] Available: http://messagelab.monash.edu.au /Nimrod

[14] S. Núnez, B. Bethwaite, J. Brenes, G. Barrantes, J. Castro, E. Malavassi, and D. Abramson, "NG-tephra: A massively parallel, nimrod/g-enabled volcanic simulation in the grid and the cloud," in Proc. the 2010 IEEE Sixth International Conference on e-Science (e-Science), pp. 129-136, 2010.

[15] R. Sharma and P. Kanungo, "Dynamic load balancing algorithm for heterogeneous multi-core processors cluster," in Proc. the 2014 Fourth International Conference on Communication Systems and Network Technologies (CSNT), 2014, pp. 288-292.

[16] European Grid Infrastructure EGI-InSPIRE. (2014). [Online] Available: http://www.egi.eu/about/egi-inspire/

[17] M. Brock and A. Goscinski, "Grids vs. clouds," in Proc. the 5th International Conference on Future Information Technology, 2010, pp. $1-6$.

[18] About the Globus Toolkit. (2014). [Online] Available: http://toolkit.globus.org /toolkit /about.html.

[19] S. M. Hashemi and A. K. Bardsiri, "Cloud computing vs. grid computing," ARPN J. Syst. Softw, vol. 2, no. 5, pp. 188-194, 2012.

[20] J. Frey, T. Tannenbaum, M. Livny, I. Foster, and S. Tuecke, "Condor-g: A computation management agent for multi-institutional grids," Cluster Computing, vol. 5, no. 3, pp. 237-246, 2002.

[21] University of Melbourne. (2014). [online] Available: http://www.cloudbus.org/middleware/

[22] Cloud-Enable Space Weather Modelling Platform. (2014). [Online] Available:

https://www.cybera.ca/assets/Completed-Projects/CESWP-Info-Sheet. pdf

[23] Welcome to TCloud. (2014). [Online]. Available: http://www.Tclouds -project.eu/

[24] About OpenNebula Project. (2014) [Online] Available: http://opennebula .org/ about/ project/

[25] Unified Cloud Interface Project. (2014). [Online]. Available: http://code.google .com/p /unifiedcloud/

[26] Cloud Monitoring. (2014). [Online]. Available: http://www.zenoss.com/solution/cloud-monitoring

[27] Enterprise Data Hub: Key to the Infor-Driven Enterprise. (2014). [Online] Available: http://www.cloudera com/content/cloudera/en/products-and-services.html.

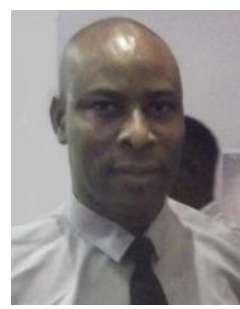

Ikechukwu Nwobodo is the director of Typical Systems Ltd UK. His past projects and work experience include: infrastructure support, information security and risk analyst, computer forensics, penetration testing and vulnerability management. He is a member of British Computer Society. He received his MSc in information security and computer forensics and $\mathrm{BSc}$ in computer network with information security system from University of East London. Has publication in various journals, conference proceedings. His research interest includes cloud computing, cloud security challenges and cyber crimes. 